\title{
A Combination Vaccine against Encephalomyocarditis Virus and Porcine Circovirus Type 2 Induces Protective Immune Response in Pigs
}

\author{
Huimin Liu1, Zhian Yin', Lu Chen², Xia Yang², Chuanqing Wang2, Hongtao Chang2* \\ ${ }^{1}$ College of Life Science, Henan Agricultural University, Zhengzhou, China \\ ${ }^{2}$ College of Animal Science and Veterinary Medicine, Henan Agricultural University, Zhengzhou, China \\ Email:1hmin01@126.com, ^ndcht@163.com
}

How to cite this paper: Liu, H.M., Yin, Z.A., Chen, L., Yang, X., Wang, C.Q. and Chang, H.T. (2018) A Combination Vaccine against Encephalomyocarditis Virus and Porcine Circovirus Type 2 Induces Protective Immune Response in Pigs. World Journal of Vaccines, 8, 30-37. https://doi.org/10.4236/wjv.2017.81004

Received: December 11, 2017

Accepted: February 25, 2018

Published: February 28, 2018

Copyright $\odot 2018$ by authors and Scientific Research Publishing Inc. This work is licensed under the Creative Commons Attribution International License (CC BY 4.0).

http://creativecommons.org/licenses/by/4.0/

\begin{abstract}
Encephalomyocarditis virus (EMCV) and porcine circovirus type 2 (PCV2) are common causative agents with high infection rate in pig farms, thus a combined vaccine against both EMCV and PCV2 is highly desirable. In the present study, we developed an oil-adjuvant combination vaccine candidate comprising of inactivated EMCV and PCV2, and evaluated the safety and immunogenicity in mice and swine. The combination vaccine was found to elicit serum antibodies and had strong neutralization activity, more importantly, passive immunization with the combined vaccine protected swine against either EMCV or PCV2 lethal infections, whereas the monovalent vaccine only prevent the one of two virus challenge. Our results demonstrated the combined vaccine was safe and induced protective immune response in mice and swine as evident from sero-conservation as well as challenge studies in swine, indicating that component vaccines did not interfere with the immunogenicity of each other.
\end{abstract}

\section{Keywords}

EMCV, PCV-2, Combination Vaccine, Safety, Immunogenicity

\section{Introduction}

Encephalomyocarditis virus (EMCV) and porcine circovirus type 2 (PCV2) are common causative agents with high infection rate in pig farms. EMCV has been recognized worldwide as a pathogen infecting a wide range of host species [1] [2] [3], and caused severe economic losses on pig production due to high mor- 
tality in piglets as a result of myocarditis and sudden death and in sows as a result of severe reproductive failure [4]. PCV2 is the major pathogen of porcine circovirus associated disease (PCVAD), which can cause severe immunosuppression in pigs and lead to secondary or other infectious diseases [5]. Both the diseases are responsible for a significant economic loss on pig farms. The frequent occurrence of mixed natural infections involving EMCV and PCV2 in pigs in Central China has been proved in our previous study, which makes clinical manifestations more complex and higher mortality. Thus, there is a potential for use of a combination vaccine for control of these infections particularly in the endemic areas.

There are no specific antiviral drugs for the treatment of these virus infections, so vaccination along with health management has been widely used to control PCV2 and EMCV in most countries. Commercial PCV2 vaccines and EMCV vaccine have been developed in some countries [6] [7], but EMCV vaccine has not been introduced into China. There are numerous PCV2 vaccine candidates in pre-clinical and clinical development [8] [9], and our group recently showed that inactivated EMCV vaccine protected mice against lethal EMCV infection (not published). With the aim of developing a combination vaccine against EMCV and PCV2 infections, we investigate in the present study the safety and immunogenicity of a combination vaccine candidate comprising of both EMCV and PCV2 inactivated whole-virus antigens. The use of combined EMCV and PCV2 vaccine will economize the production cost and vaccine application under field condition.

\section{Materials and Methods}

\subsection{Cells, Virus and Vaccine Preparation}

BHK-21 and PK-15 cells were grown in DMEM (Gibco, USA). Viruses used in this study include EMCV JZ1201 (GenBank ID: KF836386) and PCV2 Luohe (GenBank ID: EU521707) strains were isolated and identified in our laboratory. The virus were titrated was determined by the microtitration method using BHK-21 and PK-15 cells and were expressed as the 50\% tissue culture infectious dose (TCID50) according to the Reed-Muench method [10]. The EMCV (or PCV2) strain was propagated in BHK-21 cells (or PCV-free PK15 cells for the PCV2 strain) to a titer of $105 \mathrm{TCID} 50 / \mathrm{ml}$ and then treated with three freeze-thaw cycles. Virus inactivation was carried out by treating the virus-containing supernatants with a $1 / 2000$ volume of $\beta$-propiolactone. Once inactivated, the EMCV and PCV2 antigens were formulated with oil adjuvant respectively forming two monovalent oil emulsion vaccines, meanwhile, the EMCV and PCV2 were mixed together in 1:1 ratio were formulated with oil adjuvant forming a combined vaccine.

\subsection{Animals, Immunizations, ELISA, SNT and Challenge}

The animal experiments were approved by the Institutional Animal Care and 
Use Committee. Forty-eight mice female BALB/c mice (aged 6 - 8 weeks) were randomly divided into four groups ( $\mathrm{n}=12$ per group), and immunized intraperitoneally (i.p.) twice at an interval of 2 weeks. Twenty-eight pigs (30 - $40 \mathrm{~kg}, 21$ days of age), antibody-negative against EMCV and PCV2, were randomly divided into different groups (Table 1). All the animals were immunized with one of the following antigen: inactivated EMCV, inactivated PCV2, a combined vaccine comprising of EMCV and PCV2, and PBS as a negative control. The six immunized mice in each group were bled for antibody measurement, and another six mice in each group and immunized pigs were challenged with virulent strain of EMCV or PCV2 or both 14 days after the last immunization. Animals were monitored daily up to 14 days after challenge. And serum samples were collected for determining the seroconversion by indirected ELISA and by serum neutralization test (SNT). All statistical analysis was performed with GraphPad Prism software version 6.0. Neutralizing antibody titers were compared by the Student's two-tailed t-test.

\section{Results}

The immunogenicity and protective efficacy of the combined vaccine in mice were determined as shown in Figure 1(a) and Figure 1(b), the control group exhibited baseline levels of reactivity with both coating antigens; the anti-EMCV sera strongly bound to the EMCV-VP1 antigen and weakly to the PCV2-ORF2 counterpart; vice versa, the anti-PCV2 sera reacted potently with the PCV2-ORF2 coating antigen and a lesser extent with the EMCV-VP1 coating antigen. In contrast, the antisera against the bivalent vaccine exhibited high-levels of reactivity with both coating antigens. The protective efficacy of the neutralizing antisera was evaluated in vitro and in vivo. The antisera of the combined vaccine group showed potent neutralization activity against EMCV and PCV2, while monovalent vaccine groups only neutralize their respective virus (Figure 1(c) and Figure 1(d)). The results of challenge tests of immunized mice

Table 1. Experimental design showing different groups of pigs immunized with EMCV/ PCV2/combined vaccines and challenged.

\begin{tabular}{ccccc}
\hline $\begin{array}{c}\text { Group } \\
\text { (no. of animals) }\end{array}$ & Vaccine & Route & $\begin{array}{c}\text { Dose of vaccine } \\
\text { virus per pig }\end{array}$ & $\begin{array}{c}\text { Nos. protected/challenged } \\
\text { (virulent virus for challenge) }\end{array}$ \\
\hline A $(\mathrm{n}=4)$ & EMCV & i.m. & $10^{4} \mathrm{TCID} 50 / \mathrm{ml}$ & $4 / 4(\mathrm{EMCV})$ \\
$\mathrm{B}(\mathrm{n}=4)$ & PCV2 & i.m. & $10^{4} \mathrm{TCID} 50 / \mathrm{ml}$ & $4 / 4(\mathrm{PCV})$ \\
$\mathrm{C} 1(\mathrm{n}=4)$ & $\begin{array}{c}\text { Combined } \\
(\mathrm{EMCV}+\mathrm{PCV} 2) \\
\text { Combined }\end{array}$ & i.m. & $10^{4} \mathrm{TCID} 50 / \mathrm{ml}$ & $4 / 4(\mathrm{EMCV})$ \\
$\mathrm{C} 2(\mathrm{n}=4)$ & $\begin{array}{c}\text { (EMCV }+ \text { PCV2) } \\
\text { PBS }\end{array}$ & i.m. & $10^{4} \mathrm{TCID} 50 / \mathrm{ml}$ & $4 / 4(\mathrm{PCV} 2)$ \\
$\mathrm{D} 1(\mathrm{n}=4)$ & $\begin{array}{c}\text { (un-vaccined controls) } \\
\text { PBS }\end{array}$ & i.m. & $10^{4} \mathrm{TCID} 50 / \mathrm{ml}$ & $4 / 4(\mathrm{EMCV})$ \\
$\mathrm{D} 2(\mathrm{n}=4)$ & $\begin{array}{c}\text { i.m } \\
\text { (un-vaccined controls) }\end{array}$ & $10^{4} \mathrm{TCID} 50 / \mathrm{ml}$ & $4 / 4(\mathrm{PCV} 2)$ \\
$\mathrm{E}(\mathrm{n}=4)$ & $\begin{array}{c}\text { Combined } \\
\text { (EMCV }+ \text { PCV2) }\end{array}$ & i.m. & $10^{6} \mathrm{TCID} 50 / \mathrm{ml}$ & Safety test of vaccine \\
\hline
\end{tabular}




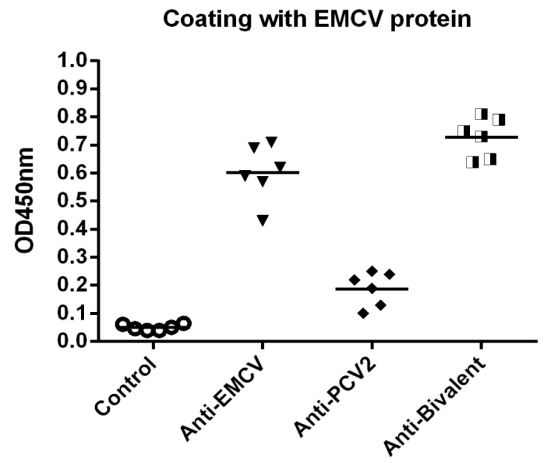

(a)

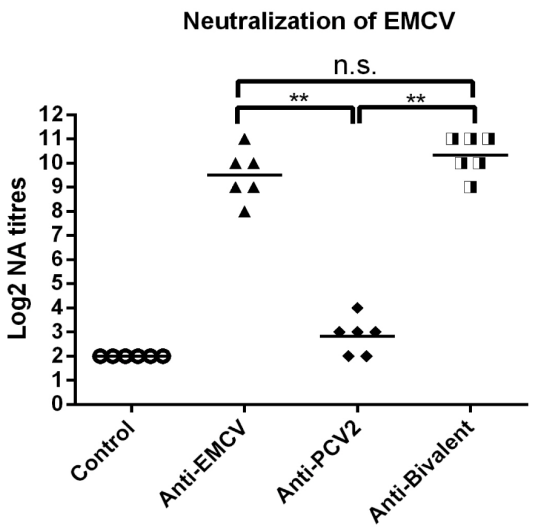

(c)

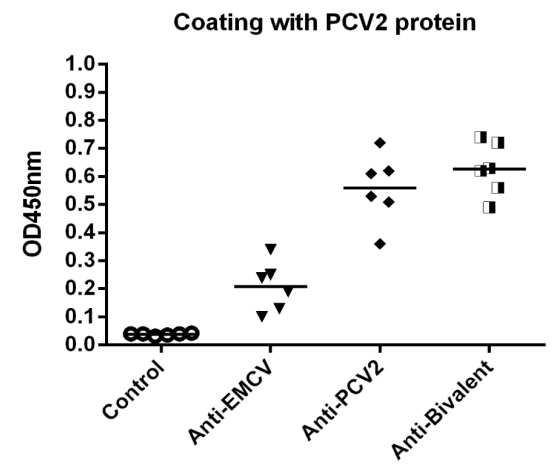

(b)

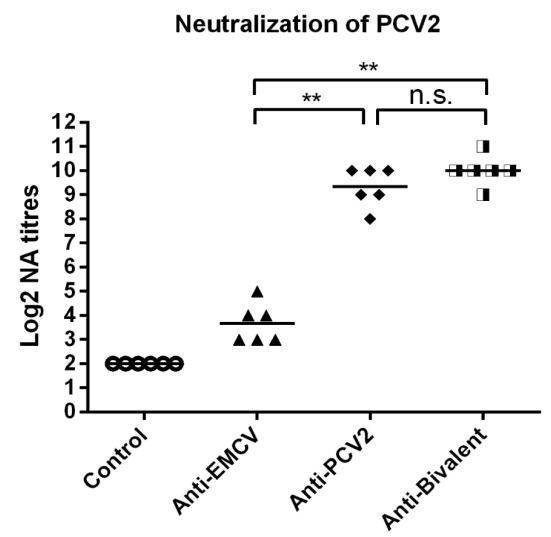

(d)

Figure 1. Antibody response in mice immunized with the inactivated vaccines. Four groups of six mice were i.p. injected with inactivated EMCV, inactivated PCV2, a combined vaccine consisting of both inactivated EMCV and PCV2, or PBS as control. Immunized mouse sera were diluted 1:100 with PBS and then used to measure virus-specific antibodies by indirected ELISA with (a) EMCV-or (b) PCV2-derived proteins as the coating antigen. Mouse serum samples collected at two weeks after the last immunization were tested for neutralization of (c) EMCV; or (d) PCV2. Neutralization titers were determined as the highest serum dilution that prevents cells from CPE. The anti-sera of the control group did not show any neutralization activity against EMCV or PCV2 at 1:8 dilution. Each symbol represents one mouse and the geometric mean value of the group. The data are representative results of two independent ELISA and neutralization experiments. Statistical significance was analyzed by Student's t test and is indicated as follows: n.s., $\mathrm{P}>0.05 ;{ }^{*}, \mathrm{p}<0.05 ;{ }^{*}, \mathrm{P}<0.005$.

showed that anti-EMCV treatment completely protected mice from EMCV infection; vice versa, anti-PCV2 treatment conferred full protection against PCV2; in contrast, the mice receiving the combined vaccine were protected from both EMCV and PCV2 infection.

The efficacy of the combined vaccine in pigs has also been evaluated, and all the immunized pigs remained apparently healthy without any adverse reactions and signs of illness on immunization with EMCV or PCV2 or combined vaccine. A marginal rise in rectal temperature $\left(39.0^{\circ} \mathrm{C} \pm 0.5^{\circ} \mathrm{C}\right)$ in all immunized pigs was noticed on days 7, 8 and 9 post-immunization (Figure 2), and then temperature returned to normal. Three pigs showed lack of appetite on day 1 post-challenge (pc), but their appetite returned to normal beginning on day $3 \mathrm{pc}$. This might consistent 


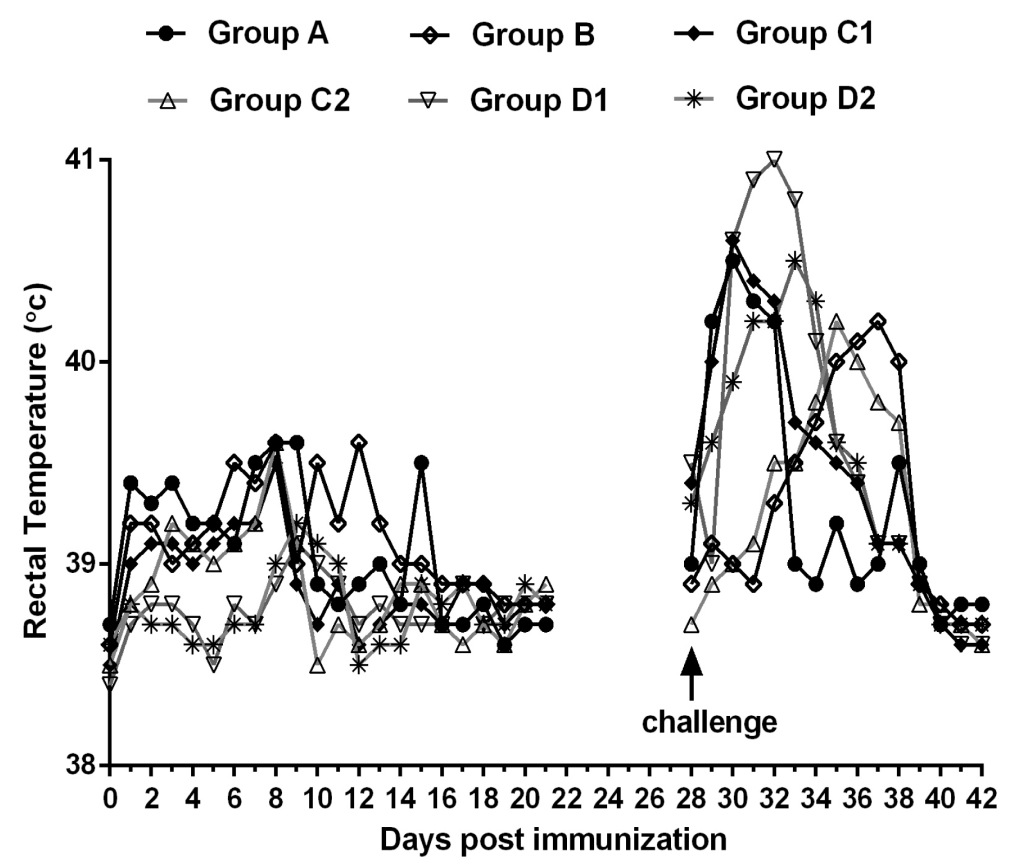

Figure 2. Temperature reaction in pigs following vaccination with EMCV or PCV2 or combined vaccines and after challenge along with non-vaccinated controls.

with the stress elicited by the immune response following vaccination and by blood collection. In addition, the protective immune response induced by the combined vaccine as evident from seroconversion as well as protection on challenge in pigs as shown in Figure 3(a) and Figure 3(b), indicating that both vaccine viruses did not interfere with the immunogenicity of each other. In addition, a sharp increase up to 14 days after vaccination was observed, which reached a plateau at around 21 days post vaccination by both indirect ELISA and SNT (Figure 3(a)). Groups A, B, C1 and C2 showed an increased production of neutralizing antibodies after the primary and secondary inoculation with monovalent and combined vaccines, and these neutralizing antibody levels were maintained at high levels for up to four weeks after the last inoculation. In contrast, swine in control groups did not produce any detectable neutralizing antibodies. No injection site reaction or side effects were observed after immunization and all swine were healthy during the immunization period.

All the pigs except group E were challenged with the respective challenge viruses. Groups A and C1 showed $100 \%$ protection on challenge with virulent EMCV. Similar findings were observed in pigs of groups B and C2 challenged with virulent PCV2. The control pigs of groups D1 and D2 showed sign of disease by their respective challenge viruses, and rise in rectal temperature was recorded up to $40^{\circ} \mathrm{C}$ (Figure 2) on day 4 th of pc. Pigs of all groups vaccinated with either EMCV, PCV2 or combined vaccines showed booster response on challenge with their respective virulent viruses. The pigs of control groups also showed rise in antibody titers on virulent virus infection, albeit to a much lesser extent than the vaccinated groups (Figure 3). 


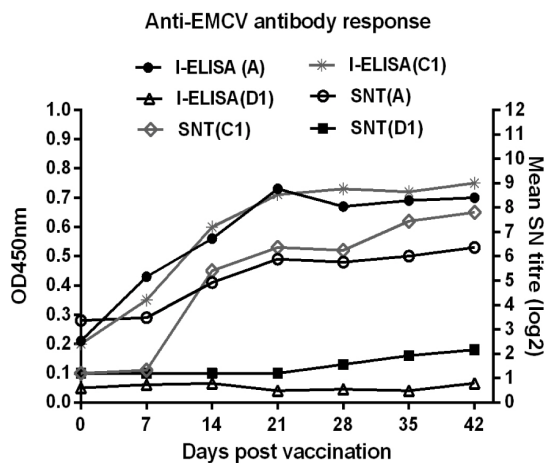

(a)

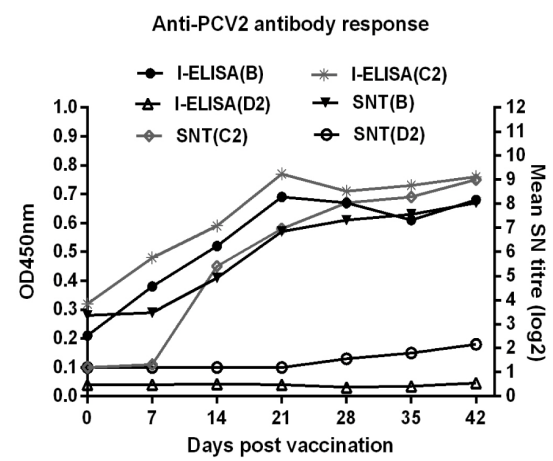

(b)

Figure 3. Development of antibodies to porcine EMCV or PCV2 (using EMCV or PCV2 inactivated vaccine/combined vaccine) as detected by Indirect-ELISA (I-ELISA) and SNT including non-vaccinated control after challenge with EMCV (a) or PCV2 (b). Details of the groups are as in Table 1.

\section{Discussion}

EMCV and PCV2 are the two major causative agents of swine, which are threatening the production of pigs in many countries and have high economic importance. In recent years, clinical survey shows that EMCV and PCV2 co-infection could result in more severe clinical symptoms and higher mortality [11]. Therefore, a vaccine that is protective against EMCV and PCV2 is critically needed. Inactivated whole-virus based EMCV or PCV2 vaccines have shown protective efficacy in animal model [12] [13] [14] [15] [16], and the results lead to a logical proposition to develop a combination vaccine consisting of inactivated EMCV and PCV2. However, formulation of bivalent or multivalent vaccines is often challenging, due to immune interference. A frequently found phenomenon is that one immunogen in a combination vaccine is immunodominant over the others, leading to unbalanced immune responses and protections against target pathogens [17]-[22]. Our results show that the combined vaccine was able to elicit serum antibodies that neutralize both EMCV and PCV2 at levels similar to that induced by the corresponding monovalent vaccines (Figure 3), indicating that no immune interference exists between two immunogens.

To the best of our knowledge, this is the first study for a combined vaccine of EMCV and PCV2, which is able to protect pigs against either EMCV or PCV2 challenge or both. PCV2 infection contributes to the severity of porcine EMCVassociated lesions when it precedes EMCV infection but not concurrent infection. However, vaccination against PCV2 alone does not decrease the potentiation of EMCV-associated lesions by PCV2. Therefore, introduction of a new efficacious combined vaccine of EMCV and PCV2 will help swine producers and practitioners reduce the number of vaccinations in growing pigs as well as control two economically important pathogens.

\section{Conflict of Interest}

The authors declare no conflicting interests. 


\section{Acknowledgements}

This research was supported by grant from the Key Scientific Research Projects of Henan province (grant 30601502).

\section{References}

[1] Gelmetti, D., Meroni, A., Brocchi, E., et al. (2006) Pathogenesis of Encephalomyocarditis Experimental Infection in Young Piglets: A Potential Animal Model to Study Viral Myocarditis. Veterinary Research, 37, 15-23. https://doi.org/10.1051/vetres:2005041

[2] Juncker-Voss, M., Prosl, H., Lussy, H., et al. (2003) Screening for Antibodies against Zoonotic Agents among Employees of the Zoological Garden of Vienna, Schonbrunn, Austria. Berliner und Munchener Tierarztliche Wochenschrift, 117, 404-409.

[3] LaRue, R., Myers, S., Brewer, L., et al. (2003) A Wild-Type Porcine Encephalomyocarditis Virus Containing a Short Poly (C) Tract Is Pathogenic to Mice, Pigs, and Cynomolgus Macaques. Journal of Virology, 77, 9136-9146. https://doi.org/10.1128/JVI.77.17.9136-9146.2003

[4] Billinis, C., Paschaleri-Papadopoulou, E., Psychas, V., et al. (1999) Persistence of Encephalomyocarditis Virus (EMCV) Infection in Piglets. Veterinary Microbiology, 70, 171-177. https://doi.org/10.1016/S0378-1135(99)00137-6

[5] Ge, X., Wang, F., Guo, X., et al. (2012) Porcine Circovirus Type 2 and Its Associated Diseases in China. Virus Research, 164, 100-106. https://doi.org/10.1016/j.virusres.2011.10.005

[6] Park, C., Jeong, J., Choi, K., et al. (2016) Efficacy of a New Bivalent Vaccine of Porcine Circovirus Type 2 and Mycoplasma hyopneumoniae (Fostera ${ }^{\mathrm{sm}} \mathrm{PCV} \mathrm{MH}$ ) under Experimental Conditions. Vaccine, 34, 270-275. https://doi.org/10.1016/j.vaccine.2015.11.034

[7] Jeoung, H.Y., Shin, B.H., Jeong, W.S., et al. (2012) A Novel Vaccine Combined with an Alum Adjuvant for Porcine Encephalomyocarditis Virus (EMCV)-Induced Reproductive Failure in Pregnant Sows. Research in Veterinary Science, 93, 1508-1511. https://doi.org/10.1016/j.rvsc.2012.02.012

[8] Pogranichniy, R.M., Yoon, K.J., Yaeger, M., et al. (2004) Efficacy of Experimental Inactivated PCV2 Vaccines for Preventing PMWS in CDCD Pigs. Proc Annu Meet Am Assoc Swine Vet, Lowa, 35, 443-444.

[9] Fraile, L., Grau-Roma, L., Sarasola, P., et al. (2012) Inactivated PCV2 One Shot Vaccine Applied in 3-Week-Old Piglets: Improvement of Production Parameters and Interaction with Maternally Derived Immunity. Vaccine, 30, 1986-1992. https://doi.org/10.1016/j.vaccine.2012.01.008

[10] Reed, L.J. and Muench, H. (1938) A Simple Method of Estimating Fifty Per Cent Endpoints. American Journal of Epidemiology, 27, 493-497. https://doi.org/10.1093/oxfordjournals.aje.a118408

[11] Chang, H.T., Liu, H.M., Li, Y.T., et al. (2016) Analysis of Pathogenicity in Piglets Concurrent Infection with Encephalomyocarditis Virus under Immunosuppression Caused by Porcine Circovirus Type 2. Acta veterinaria et zootechnica Sinica, 47, 1435-1442.

[12] Hunter, P., Swanepoel, S.P., Esterhuysen, J.J., et al. (1998) The Efficacy of an Experimental Oil-Adjuvanted Encephalomyocarditis Vaccine in Elephants, Mice and Pigs. Vaccine, 16, 55-61. https://doi.org/10.1016/S0264-410X(97)00158-8 
[13] McLelland, D.J., Kirkland, P.D., Rose, K.A., Dixon, R.J. and Smith, N. (2005) Serologic Responses of Barbary Sheep (Ammotragus lervia), Indian Antelope (Antilope cervicapra), Wallaroos (Macropus robustus), and Chimpanzees (Pan troglodytes) to an Inactivated Encephalomyocarditis Virus Vaccine. Journal of Zoo and Wildlife Medicine, 36, 69-73. https://doi.org/10.1638/03-054

[14] Chae, C. (2012) Commercial Porcine Circovirus Type 2 Vaccines: Efficacy and Clinical Application. The Veterinary Journal, 194, 151-157.

https://doi.org/10.1016/j.tvjl.2012.06.031

[15] Seo, H.W., Han, K., Kim, D., et al. (2011) Effects of an Inactivated Porcine Circovirus Type 2 (PCV2) Vaccine on PCV2 Virus Shedding in Semen from Experimentally Infected Boars. Clinical and Vaccine Immunology, 18, 1091-1096. https://doi.org/10.1128/CVI.05027-11

[16] Yang, K., Li, W., Niu, H., et al. (2012) Efficacy of Single Dose of an Inactivated Porcine Circovirus Type 2 (PCV2) Whole-Virus Vaccine with Oil Adjuvant in Piglets. Acta Veterinaria Scandinavica, 54, 67. https://doi.org/10.1186/1751-0147-54-67

[17] Patriarca, P.A., Wright, P.F. and John, T.J. (1991) Factors Affecting the Immunogenicity of Oral Poliovirus Vaccine in Developing Countries: Review. Reviews of Infectious Diseases, 13, 926-939. https://doi.org/10.1093/clinids/13.5.926

[18] Guy, B., Barban, V., Mantel, N., Aguirre, M., Gulia, S., Pontvianne, J., et al. (2009) Evaluation of Interferences between Dengue Vaccine Serotypes in a Monkey Model. The American Journal of Tropical Medicine and Hygiene, 80, 302-311.

[19] Anderson, K.B., Gibbons, R.V., Edelman, R., Eckels, K.H., Putnak, R.J., Innis, B.L., et al. (2011) Interference and Facilitation between Dengue Serotypes in a Tetravalent Live Dengue Virus Vaccine Candidate. The Journal of Infectious Diseases, 204, 442-450. https://doi.org/10.1093/infdis/jir279

[20] Sabchareon, A., Lang, J., Chanthavanich, P., Yoksan, S., Forrat, R., Attanath, P., et al. (2002) Safety and Immunogenicity of Tetravalent Live-Attenuated Dengue Vaccines in Thai Adult Volunteers: Role of Serotype Concentration, Ratio, and Multiple Doses. The American Journal of Tropical Medicine and Hygiene, 66, 264-272. https://doi.org/10.4269/ajtmh.2002.66.264

[21] Sun, W., Edelman, R., Kanesa-Thasan, N., Eckels, K.H., Putnak, J.R., King, A.D., et al. (2003) Vaccination of Human Volunteers with Monovalent and Tetravalent Live Attenuated Dengue Vaccine Candidates. The American Journal of Tropical Medicine and Hygiene, 69, 24-31. https://doi.org/10.4269/ajtmh.2003.69.6_suppl.0690024

[22] Kitchener, S., Nissen, M., Nasveld, P., Forrat, R., Yoksan, S., Lang, J., et al. (2006) Immunogenicity and Safety of Two Live-Attenuated Tetravalent Dengue Vaccine Formulations in Healthy Australian Adults. Vaccine, 24, 1238-1241.

https://doi.org/10.1016/j.vaccine.2005.09.029 